\title{
Clostridium difficile Toxin A Perturbs Cytoskeletal Structure and Tight Junction Permeability of Cultured Human Intestinal Epithelial Monolayers
}

\author{
Gail Hecht, Charalabos Pothoulakis, J. Thomas LaMont, and James L. Madara \\ Departments of Pathology (Gastrointestinal Pathology), Brigham and Women's Hospital and Harvard Medical School, Boston, \\ Massachusetts 02115; Department of Gastroenterology, Brigham and Women's Hospital, Boston, Massachusetts 02115; \\ Harvard Digestive Diseases Center, Boston, Massachusetts 02115; and Section of Gastroenterology, Evans Memorial \\ Department of Clinical Research, Boston University School of Medicine, Boston, Massachusetts 02118
}

\begin{abstract}
Toxin A of Clostridium difficile causes severe inflammatory enterocolitis in man and animals that appears to be mediated in part by acute inflammatory cells that migrate into the toxin A-exposed mucosa. To determine the direct effects of toxin A on intestinal epithelial permeability and structure in the absence of other modulating factors, we used cultured monolayers of a human intestinal epithelial cell line $\left(T_{84}\right)$. $A$ toxin $A$ concentration of $7 \times 10^{-1} \mu \mathrm{g} / \mathrm{ml}\left(3 \times 10^{-9} \mathrm{M}\right)$ nearly abolished monolayer transepithelial resistance within 6-8 h. This marked permeability defect occurred while the monolayers were still confluent. Dual sodium-mannitol flux studies localized the permeability defect to the intercellular tight junction. Cytotoxicity assays and morphological evaluation using Nomarski optics and electron microscopy failed to demonstrate any evidence of cell damage at the time the maximum resistance response was observed. Fluorescent staining for $F$ actin, however, revealed a marked decrease in fluorescent intensity in toxin-treated monolayers versus controls. These data show that toxin A can directly affect the barrier function of this model intestinal epithelium and initially does so by selectively enhancing tight junction permeability. Furthermore, cytoskeletal structure is markedly altered over the same time course, although the integrity of individual cells is maintained. Because the cytoskeleton of intestinal epithelial cells is known to be capable of regulating tight junction permeability, we speculate that the above effects of toxin $A$ on epithelial barrier function result from alterations of the cytoskeleton.
\end{abstract}

\section{Introduction}

Toxigenic strains of the anaerobic bacterium Clostridium difficile are the major cause of antibiotic-associated pseudomembranous colitis. $C$. difficile produces two protein exotoxins that appear to be responsible for this disease. Toxin A, an enterotoxin, elicits severe epithelial damage associated with hemorrhage and fluid secretion when injected into rabbit ileal loops $(1,2)$. Toxin $B$, on the other hand, does not cause mucosal injury but does induce alterations in the shape of cul-

Address reprint requests to Dr. James L. Madara, Department of Pathology, Brigham and Women's Hospital, 20 Shattuck Street, Room 1423, Boston, MA 02115.

Received for publication 2 February 1988 and in revised form $1 \mathrm{July}$ 1988.

J. Clin. Invest.

(c) The American Society for Clinical Investigation, Inc.

0021-9738/88/11/1516/09 \$2.00

Volume 82, November 1988, 1516-1524 tured mesenchymal cells $(3,4)$ and is therefore termed a cytotoxin.

It is unclear which of the multiple populations of cells in the intestinal mucosa (epithelial, endothelial, inflammatory cells, etc.) is primarily affected by $C$. difficile toxins. Based on analysis of human rectal biopsies displaying pseudomembranous colitis in the early phase, Price and Davies (5) speculated that the initial target might be the endothelium lining the superficial capillary network. In this view, the epithelial effects would be a secondary event. Recent comparative studies of the effects of highly purified toxin A on either in vivo intestinal loops or mucosal explants have led to speculation that toxin A-induced release of mediators from inflammatory cells might lead to secondary injury to the intestinal epithelium (6). Toxin A has been shown to directly affect one type of nonintestinal epithelial cell. Rothman et al. (7) demonstrated that toxin A inhibited protein synthesis in HeLa cell cultures. However, the direct relevance of this observation to the defects in mucosal structure and function seen in pseudomembranous colitis is unclear.

To determine if toxin A exerted any direct effects on intestinal epithelial cells, we applied highly purified toxin A to monolayers composed of a human intestinal epithelial cell line $\left(\mathrm{T}_{84}\right)$. These monolayers, which consist of confluent, polarized, columnar cells develop high, stable resistances to passive ion flow $(8,9)$. The particular advantage of this approach is that human intestinal epithelial cells devoid of any mesenchymal, vascular, or inflammatory components are used. These studies demonstrate that $C$. difficile toxin A directly alters epithelial permeability without producing cell death or disrupting the confluence of the monolayer. Further analysis reveals that this increase in permeability can be solely accounted for by a paracellular (tight junction) effect. Lastly, this permeability abnormality is paralleled by dramatic alterations in that portion of the cytoskeleton thought to be important in regulation of tight junction permeability.

\section{Methods}

Cell culture. $T_{84}$ cells were grown, passaged, and mounted as previously described (8-10) on either collagen-coated filters, 24-well plates, or glass coverslips. Transepithelial resistances generated by the ringmounted monolayers used for these studies ranged from 400 to 1,700 $\Omega \cdot \mathrm{cm}^{2}$. Such resistance variation is attributable to monolayer-tomonolayer variation in tight junction permeability (10). Passages 26-37 were established from cells obtained from K. Dharmsathaphorn (University of California, San Diego, San Diego, CA) and passages 55-70 were established from cells obtained from American Type Culture Collection (Rockville, MD).

Purification of toxin A. Toxin A was purified to homogeneity (single band at $229 \mathrm{kD}$ by SDS-PAGE) from broth culture supernatants of 
toxigenic strains of $C$. difficile according to the methods of Sullivan et al. (11). Enterotoxin activity was assayed using the ligated ileal loop assay (12). Toxin A preparations used in this study exhibited cytotoxicity in the fibroblast assay at a $10^{-3}-10^{-4}$ dilution as previously reported $(1,11)$. The purified toxin was diluted in Ringer's solution to a final concentration of $50 \mu \mathrm{g} / \mathrm{ml}$ and stored at $-70^{\circ} \mathrm{C}$ in aliquots sufficient for one experiment. For experimental use, the toxin was thawed and diluted to the desired concentration in cell culture medium, then filtered through a $0.2-\mu \mathrm{m}$ filter to ensure sterility. In most cases, the toxin was thawed and diluted immediately before use. However, a bioassay of the toxin (effect on monolayer resistance as reported here) determined that it was effective for at least $11 \mathrm{~d}$ after thawing when stored at $4^{\circ} \mathrm{C}$. The results we describe cannot be attributed to contaminating endotoxins, as the barrier function of $\mathrm{T}_{84}$ cells is resistant to endotoxins (Madara, J. L., and G. Hecht, unpublished observations).

Toxin incubation with $T_{84}$ cells. $T_{84}$ cells grown in cell culture medium were allowed to reach confluence as determined by phase microscopy, if on plastic or glass substrates, or by development of a high, stable resistance $\left(400-1,700 \Omega \cdot \mathrm{cm}^{2}\right)$ if cultured on ringmounted, collagen-coated filters. At the time of experimentation, tissue culture medium was decanted and replaced with either toxin-containing or toxin-free medium. Toxin was applied only to the apical (mucosal) surface of monolayers unless stated otherwise.

Flux studies. Modified low-turbulence Ussing chambers were used for flux studies as previously described $(8,13)$. Transepithelial flux rates for sodium, mannitol, raffinose, and inulin were determined in 39 monolayers as previously outlined $(8,13)$.

To measure toxin-elicited mannitol efflux from $T_{84}$ cells (to ascertain if toxin $\mathrm{A}$ induced a plasma membrane mannitol leak), cells were trypsinized in the presence of $1 \mu \mathrm{Ci} / \mathrm{ml}\left[{ }^{3} \mathrm{H}\right]$ mannitol and grown to confluency in 24-well plates in media containing the same concentration of labeled mannitol. After four washes in PBS, cells were exposed to toxin-free or toxin-containing media. Supernatant was collected at a time corresponding to the maximum toxin-elicited resistance drop ( $\sim 90 \%$ decrease from baseline). Mannitol released into the supernatant was then measured by scintillation counting. That intracellular labeling had occurred was confirmed by the finding that disruption of cells with $0.1 \%$ Triton X-100 released large quantities of tracer.

Sterile chamber resistance measurements. Transepithelial resistances of $T_{84}$ monolayers were measured under sterile conditions using a chamber designed by Dr. Roderick Steele (Laboratory of Technical Development, National Institutes of Health, Bethesda, MD) who kindly provided us with the technical drawings and allowed us to reproduce it. This "Steele" chamber was operated similarly to the Ussing chamber. Empirical tests using artificial membranes showed that the sterile chamber resistance measurements closely agreed with those obtained in Ussing chambers. It was also demonstrated that control monolayers could be removed from and reinserted into the sterile chamber to obtain serial measurements at intervals of at least $2 \mathrm{~h}$ without a significant change in resistance, thus allowing each monolayer to serve as its own control.

Lactate dehydrogenase $(\mathrm{LDH})^{1}$ and ${ }^{51} \mathrm{Cr}$ release. $\mathrm{LDH}$ assays were performed by incubating confluent monolayers with $1 \mathrm{ml}$ of toxincontaining or toxin-free medium for $8 \mathrm{~h}$, at which time $0.5 \mathrm{ml}$ of medium was collected from each well and analyzed for LDH (14). At the end of the experiment, total LDH concentration was determined by adding $1 \mathrm{ml}$ of $0.1 \%$ Triton X-100 to the wells for $10 \mathrm{~min}$, then repeatedly pipetting the contents of each well to ensure lysis of all cells. $0.5 \mathrm{ml}$ of this homogenate was collected from each well and assayed for $\mathrm{LDH}$.

For ${ }^{51} \mathrm{Cr}$-release assays, confluent monolayers of $\mathrm{T}_{84}$ cells grown on 24-well tissue culture plates were loaded with ${ }^{51} \mathrm{Cr}$ by incubating the cells in each well with $30 \mu \mathrm{Ci}$ for $18 \mathrm{~h}$, at which time excess ${ }^{51} \mathrm{Cr}$ was removed by washing each well three times with PBS. The protocol

1. Abbreviations used in this paper: EM, electron micrograph; LDH, lactate dehydrogenase. outlined above for the $\mathrm{LDH}$ assay was then followed and ${ }^{51} \mathrm{Cr}$ concentrations were determined by scintillation counting. Total ${ }^{51} \mathrm{Cr}$ release was determined by adding $1 \mathrm{ml}$ of $0.1 \%$ Triton X-100 to the monolayer for $10 \mathrm{~min}$, pipetting, and counting an $0.5-\mathrm{ml}$ aliquot.

Morphology. Immediately after removal from either the sterile or Ussing chamber, the monolayers were placed in $2 \%$ glutaraldehyde in $0.1 \mathrm{M} \mathrm{Na}$ cacodylate buffer and processed for thick sectioning as previously described $(8,9)$.

Fluorescent staining of $\mathrm{F}$ actin was performed using rhodamine-labeled phalloidin (Molecular Probes, Eugene, OR). Monolayers were grown on glass coverslips to confluency before toxin A was added. After the indicated incubation period, cell-coated coverslips were rinsed in PBS, fixed for $10-15 \mathrm{~min}$ at room temperature in $3.7 \%$ formaldehyde, rinsed again in PBS, permeabilized for $5 \mathrm{~min}$ in $-20^{\circ} \mathrm{C}$ acetone, air dried, stained with rhodamine-labeled phalloidin for 30 min at room temperature in the dark, rinsed twice in PBS, and mounted upside down on microscope slides in a PBS-glycerol-p-phenylendiamine solution to preserve fluorescence. Cells were then examined and photographed using a Zeiss fluorescent microscope. To compare the degree of labeling between toxin-treated and control monolayers, photomicrographs were taken with Kodak Tri-X Pan film (ASA $1,000)$ using standard preset exposure and development times. To compare the pattern of labeling between toxin-treated and control monolayers, exposures were varied to optimize the resulting images.

\section{Results}

Effects of toxin $A$ concentration on epithelial resistance. The effect of varying concentrations of $C$. difficile toxin A on epithelial resistance (a measure of permeability to ions) was first examined after $72 \mathrm{~h}$ of continuous toxin exposure. Doses of toxin A ranging from $7 \times 10^{-4}$ to $7 \mu \mathrm{g} / \mathrm{ml}$ were evaluated. Over this time course, doses of $7 \times 10^{-2}$ to $7 \mu \mathrm{g} / \mathrm{ml}$ nearly abolished transepithelial resistance $(87 \pm 15.5,98 \pm 1.4$, and $99 \pm 1.1 \%$ resistance fall respectively, compared with controls, Fig. 1). The lowest two doses, $7 \times 10^{-4}$ and $7 \times 10^{-3} \mu \mathrm{g} / \mathrm{ml}$, caused a slight increase in resistance, although this observation was not further pursued (Fig. 1).

Additional studies were then performed after a $24-\mathrm{h}$ incubation with toxin concentrations of $7 \times 10^{-1}, 3.5 \times 10^{-1}, 1.75$ $\times 10^{-1}$, and $7 \times 10^{-2} \mu \mathrm{g} / \mathrm{ml}$. The highest dose again diminished resistance by $99 \pm 0.3 \% ; 3.5 \times 10^{-1} \mu \mathrm{g} / \mathrm{ml}$ decreased resistance by $83 \pm 11 \% ; 1.75 \times 10^{-1} \mu \mathrm{g} / \mathrm{ml}$ lowered resistance by $64 \pm 18 \%$, whereas the lowest dose resulted in a mean reduction of only $13.5 \pm 9.5 \%$. A toxin concentration of $7 \times 10^{-1} \mu \mathrm{g} / \mathrm{ml}(3$ $\times 10^{-9} \mathrm{M}$ ) was used for the remainder of the experiments.

Polarity of resistance response. In the above dose-response studies, toxin A was added only to the mucosal surface of $T_{\mathbf{8 4}}$ monolayers. To determine if this response exhibited polarity, a toxin concentration known to nearly ablate transepithelial resistance by $24 \mathrm{~h}\left(7 \times 10^{-1} \mu \mathrm{g} / \mathrm{ml}\right)$ was applied only to the serosal side of confluent monolayers. In this set of experiments, each monolayer served as its own control as resistance values were followed serially in the sterile chamber. $16 \mathrm{~h}$ after the application of toxin $A$ to the serosal side of $\mathrm{T}_{84}$ monolayers, the resistance had decreased by $84 \pm 6.7 \%$, while the resistances of control monolayers had dropped by only $6 \pm 5.7 \%$ (Fig. 2), demonstrating that the effect of toxin $A$ on resistance was not dependent on apical exposure.

We feel confident that the observed resistance drop could not be accounted for by diffusion of toxin molecules to the mucosal side because toxin $A$ is a large protein $(230 \mathrm{kD}$, reference 6 ) and our previous findings indicate that confluent $T_{84}$ 


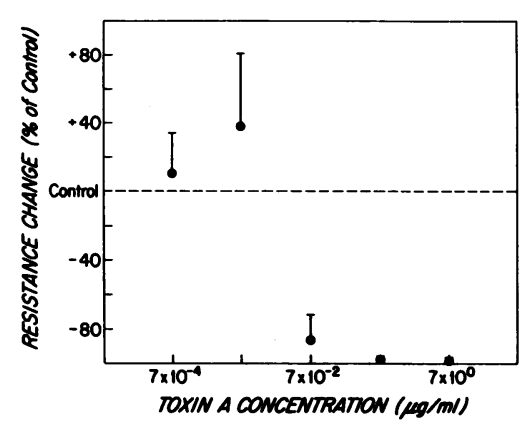

Figure 1. Effects of varying doses of $C$. difficile toxin A on transepithelial resistance of $\mathrm{T}_{84}$ monolayers after a 72-h incubation period. Confluent $\mathrm{T}_{84}$ monolayers on ring-mounted, collagen-coated filters were placed on glass beads in plastic tissueculture wells. $C$. difficile toxin $\mathrm{A}$ at concentra-

tions ranging from $7 \times 10^{-4}$ to $7 \mu \mathrm{g} / \mathrm{ml}$ was placed on the apical surface of the monolayers and incubated for $72 \mathrm{~h}$ at $37^{\circ} \mathrm{C}$ in a $95 \%$ air $/ 5 \% \mathrm{CO}_{2}$ atmosphere. Monolayer resistances were then measured in Ussing chambers. Resistance changes (percent rise or fall compared with controls) are expressed as $\mathrm{x} \pm$ SEM from three separate experiments. Maximum resistance falls were seen with the three highest concentrations, whereas the lowest two doses actually appeared to induce a resistance rise.

monolayers show minimal permeability to molecules as small as $5 \mathrm{kD}(8)$.

Time course of the resistance response. Time course studies revealed that monolayer resistance had decreased only slightly after $2 \mathrm{~h}$ of exposure to $7 \times 10^{-1} \mu \mathrm{g} / \mathrm{ml}$ toxin $\mathrm{A}(11.9 \pm 9.8 \%)$, but by $4 \mathrm{~h}$ had fallen $69.8 \pm 4.56 \%$ and by $8 \mathrm{~h}$ was nearly ablated (89.2 $\pm 1.8 \%$ resistance fall, Fig. 3$)$.

$L D H$ and ${ }^{51} \mathrm{Cr}$-release assays. To determine whether the observed toxin A-induced decrease in resistance was the result

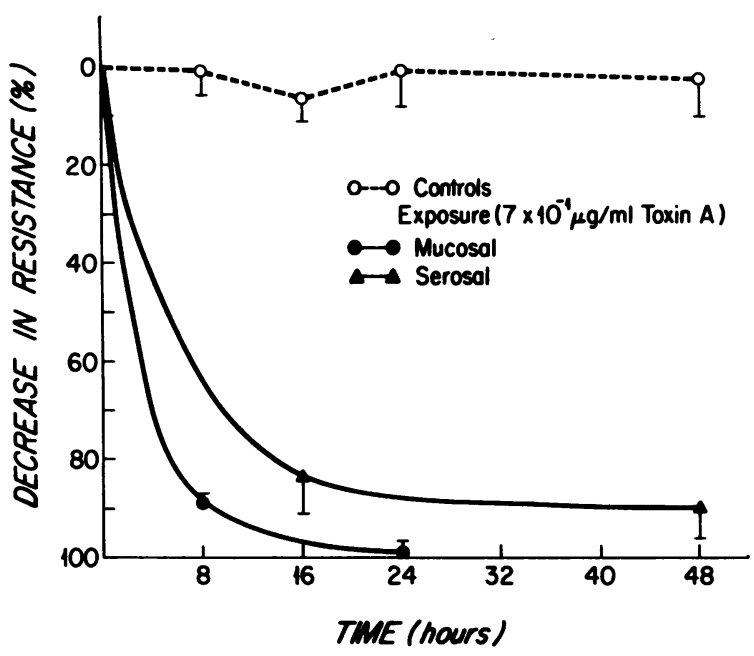

Figure 2. Effect of serosal exposure of $C$. difficile toxin $\mathrm{A}$ on $\mathrm{T}_{84}$ transepithelial resistances. Confluent $\mathrm{T}_{\mathbf{8 4}}$ monolayers on ringmounted, collagen-coated filters were placed on glass beads in plastic tissue-culture wells, such that both the mucosal and serosal surfaces had access to medium. $7 \times 10^{-1} \mu \mathrm{g} / \mathrm{ml}$ of toxin $\mathrm{A}$ was placed in the serosal medium only and monolayers were incubated under standard tissue culture conditions. Transepithelial resistances were measured in the sterile chamber $24 \mathrm{~h}$ before and 16 and $48 \mathrm{~h}$ after the addition of toxin A. After 16 and $48 \mathrm{~h}$ of toxin exposure to the serosal surface of the monolayers, transepithelial resistance had dropped $84 \pm 6.7$ and $90 \pm 10 \%$, respectively, whereas resistances of control monolayers remained relatively stable $(6 \pm 5.7$ and $2.5 \pm 8.1 \%$ resistance drop, respectively). $n=4-6$ for each data point.

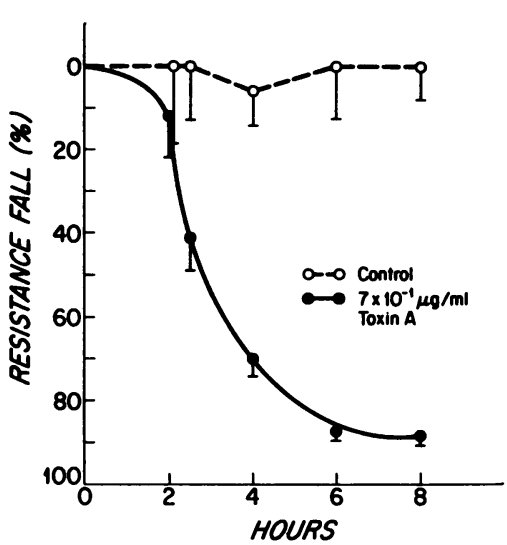

Figure 3. Time course of resistance response of $T_{84}$ monolayers to 7 $\times 10^{-1} \mu \mathrm{g} / \mathrm{ml}$ of mucosally applied $C$. difficile toxin A. Only a slight fall in resistance was observed after $2 \mathrm{~h}$ of toxin exposure (11.9 $99.8 \%)$, whereas by $6 \mathrm{~h}$, the resistance response was near maximal, resistances having fallen by $87.5 \pm 2.1 \%$. Each data point represents an $n$ of 3-6.

of cell death, $\mathrm{LDH}$ and ${ }^{51} \mathrm{Cr}$-release assays were performed. Incubation of monolayers with $7 \times 10^{-1} \mu \mathrm{g} / \mathrm{ml}$ toxin $A$ for $8 \mathrm{~h}$, conditions that elicited large changes in resistance (and mannitol flux, see below), did not increase LDH release over control values, as shown in Table $\mathrm{I}$. A ${ }^{51} \mathrm{Cr}$-release assay was performed in an identical manner and revealed that the disintegrations per minute from toxin-treated versus control cells were also not significantly different $\left(1.97 \times 10^{5} \pm 1.4 \times 10^{4}\right.$ and $1.71 \times 10^{5} \pm 1.1 \times 10^{4}$, respectively, $P>0.05$, Table I). The decreased resistances observed in $\mathrm{T}_{\mathbf{8 4}}$ monolayers after an 8-h exposure to $7 \times 10^{-1} \mu \mathrm{g} / \mathrm{ml}$ of toxin A cannot, therefore, be attributed to cell death. These data furthermore indicate that toxin A did not cause the plasma membranes of epithelial cells to become leaky to small molecules.

Flux studies. To define the site of the permeability defect (transcellular versus paracellular) caused by $C$. difficile toxin A on $\mathrm{T}_{84}$ monolayers, serosal-to-mucosal dual sodium-mannitol flux studies were performed. We used the fact that once the toxin A-induced resistance decline began, the continual presence of toxin A was not required for the full effect to be expressed (data not shown). Toxin $A$ at a concentration of 7 $\times 10^{-1} \mu \mathrm{g} / \mathrm{ml}$ thus was applied to the apical surface of monolayers previously determined to be in the high-resistance state. The monolayers were incubated for $2 \mathrm{~h}$ in the presence of toxin $A$, then inserted into toxin-free solution in Ussing chambers. The flux rates of both $\mathrm{Na}^{+}$and mannitol were then determined for eight subsequent 30 -min periods and were plotted against each other (Fig. 4). The slope of this relation-

Table I. Effect of Toxin A on LDH and ${ }^{51}$ Cr Release After $8 h$ Continuous Incubation

\begin{tabular}{lcc}
\hline & LDH & ${ }^{\text {s1 } C r}$ \\
\hline & U/liter & dpm $/$ well \pm SEM \\
Toxin A $\left(7 \times 10^{-1} \mu g / m l\right)$ & 104 & $1.97 \times 10^{5} \pm 1.4 \times 10^{4}$ \\
Control & 116 & $1.71 \times 10^{5} \pm 1.1 \times 10^{4}$ \\
Control after Triton X-100 & 960 & $1.28 \times 10^{6} \pm 5.5 \times 10^{4}$
\end{tabular}

Results are from 12 control and 12 toxin-treated monolayers ( 7 $\times 10^{-1} \mu \mathrm{g} / \mathrm{ml}$ ). LDH values were obtained from six pooled experimental monolayers and six pooled control monolayers. ${ }^{51} \mathrm{Cr}$-release data were obtained from six control and six toxin-treated monolayers. ${ }^{51} \mathrm{Cr}$ release after toxin exposure was not significantly different than control values $(P>0.05)$. 


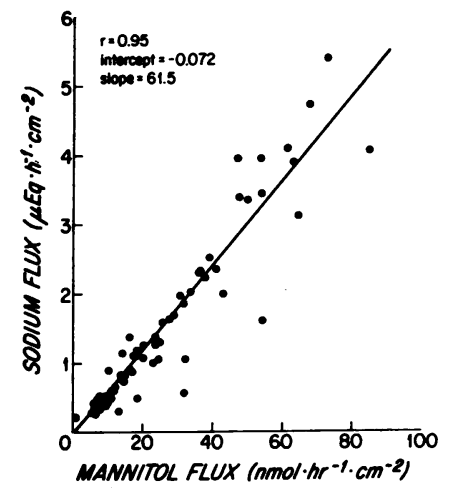

points obtained from control monolayers ( 28 total) reside below a sodium flux of $0.6 \mu \mathrm{eq} \times \mathrm{h}^{-1} \times \mathrm{cm}^{-2}$. Only two points from toxin-exposed monolayers are located in this area.

ship was $61.5(r=0.95)$. This result is extremely close to that calculated for a permeability effect solely attributable to altered tight junction permeability (predicted slope $=54$, calculated on basis of diffusion coefficients and concentrations of $\mathrm{Na}^{+}$and mannitol, see references 15 and 16). If data points obtained from monolayers in the preperturbed state were excluded from such calculations, the slope was 62.0 and $r$ $=0.93$. It can therefore be concluded that the toxin A-induced permeability defect is primarily a tight junction effect rather than a transcellular effect, in which case the increase in sodium flux would far exceed that of mannitol (15).

Mannitol efflux experiments were performed at a time when resistance was maximally decreased and transepithelial mannitol flux was increased severalfold. Toxin A did not significantly enhance leak of mannitol across plasma membranes as indicated by mannitol efflux $(944 \pm 56$ vs. $1,060 \pm 110 \mathrm{dpm} /$ well, control vs. toxin A-exposed, respectively, $P>0.05$ ).

To ascertain the approximate size of the tight junction barrier defect elicited by toxin $\mathrm{A}$, unidirectional fluxes were

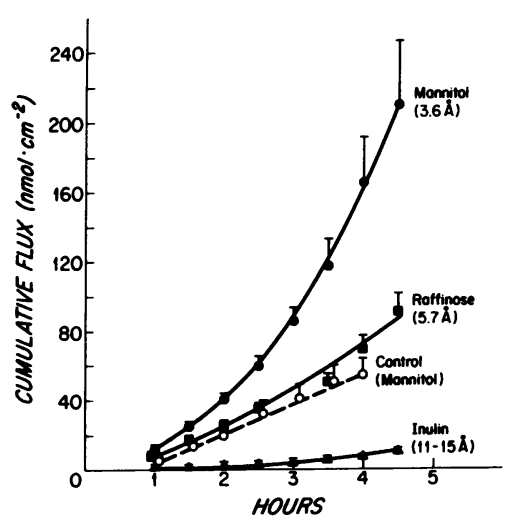

Figure 5. Comparison of cumulative flux rates of variously sized molecules. $\mathrm{T}_{84}$ monolayers were preincubated with $7 \times 10^{-1} \mu \mathrm{g} / \mathrm{ml}$ of toxin A before insertion into toxin-free Ussing chambers. The permeability defect induced by toxin $\mathrm{A}$ after $6 \mathrm{~h}$ allowed small molecules, such as mannitol (3.6

$\AA$ ), to traverse the paracellular space yet the monolayer was still able to provide barrier function as effective as that of control monolayers against the passage of molecules such as raffinose (5.7 $\AA$ ) and inulin $(11.5 \AA)(P>0.05)$. A total of 39 monolayers were used for this set of experiments ( 26 experimental and 13 controls). The linear nature of the plot for mannitol permeability in control monolayers over time versus the progressively steeper slope produced by toxin-treated monolayers indicates that toxin-induced defects increased over time, whereas the mannitol permeability of control monolayers was unchanged.
Table II. Effect of C. difficile Toxin A on Flux Rates of Three Solutes

\begin{tabular}{lcc}
\hline & \multicolumn{2}{c}{ Flux at 4 hours } \\
\cline { 2 - 3 } \multicolumn{1}{c}{ Molecule } & Control & Experimental \\
\hline \multicolumn{1}{c}{ Size, $A$} & & $n m o l \times h^{-1} \times \mathrm{cm}^{-2} \pm S E M$ \\
Mannitol (3.6) & $6.2 \pm 1.2$ & $47.9 \pm 10.0(P<0.005)$ \\
Raffinose (5.7) & $5.9 \pm 1.8$ & $8.6 \pm 1.6 \quad(P>0.05)$ \\
Inulin (11.5) & $0.41 \pm 0.14$ & $1.3 \pm 0.33(P>0.05)$ \\
& & \\
\hline
\end{tabular}

A total of 39 monolayers were used for this set of experiments (26 experimental and 13 controls).

performed on control and toxin-treated monolayers using $\left[{ }^{3} \mathrm{H}\right]$ mannitol (3.6 $\AA$ hydrodynamic radius), $\left[{ }^{3} \mathrm{H}\right]$ raffinose $(5.7$ $\AA$ ), and $\left[{ }^{14} \mathrm{C}\right]$ inulin (11.5 $\AA$ ). The cumulative flux of each molecule plotted against time is shown in Fig. 5. Molecules the size of mannitol (3.6 $\AA$ ) readily traversed the paracellular space of toxin-treated monolayers relative to those molecules two to four times larger (Fig. 5). This figure also includes the data obtained for mannitol fluxes in control monolayers so that the changing slope of the mannitol data after toxin exposure can be appreciated (see below). In the last flux period shown, the relative rates of flux across toxin exposed monolayers for mannitol, raffinose, and inulin were 47.9 $\pm 10.0,8.6 \pm 1.6$, and $1.3 \pm 0.33 \mathrm{nmol} \times \mathrm{h}^{-1} \times \mathrm{cm}^{-2}$, respectively. As can be seen from the data in Table II, the fluxes of raffinose and inulin in control and toxin-treated monolayers over the 4-h experiment were not significantly different $(P>0.05)$, whereas toxin elicited large changes in mannitol flux. These data confirm the assays that indicated the absence of cell death, because they show that the defects in the epithelial barrier elicited by toxin A are exceedingly small.

Lastly, as seen in Fig. 5, the mannitol-permeable sites accumulate at an increasing rate over the 4-h period after toxin A exposure.

The above data indicate that toxin A exposure elicits a change in the radius of the transjunctional pores (i.e., the per-

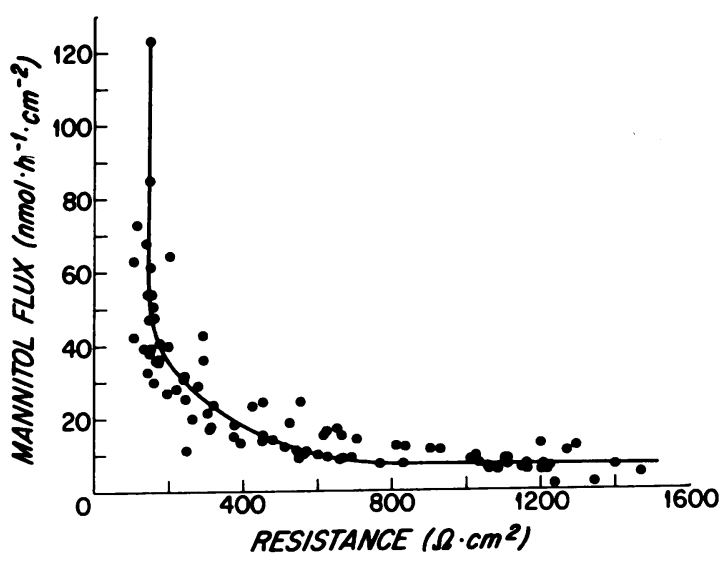

Figure 6. Paracellular serosal-to-mucosal flux rates of mannitol versus resistance. Resistance is a much more sensitive indicator of barrier function than mannitol flux, as major changes in resistance reflect only minor alterations in mannitol flux. Large increments in mannitol flux occurred only after resistance was maximally affected. 

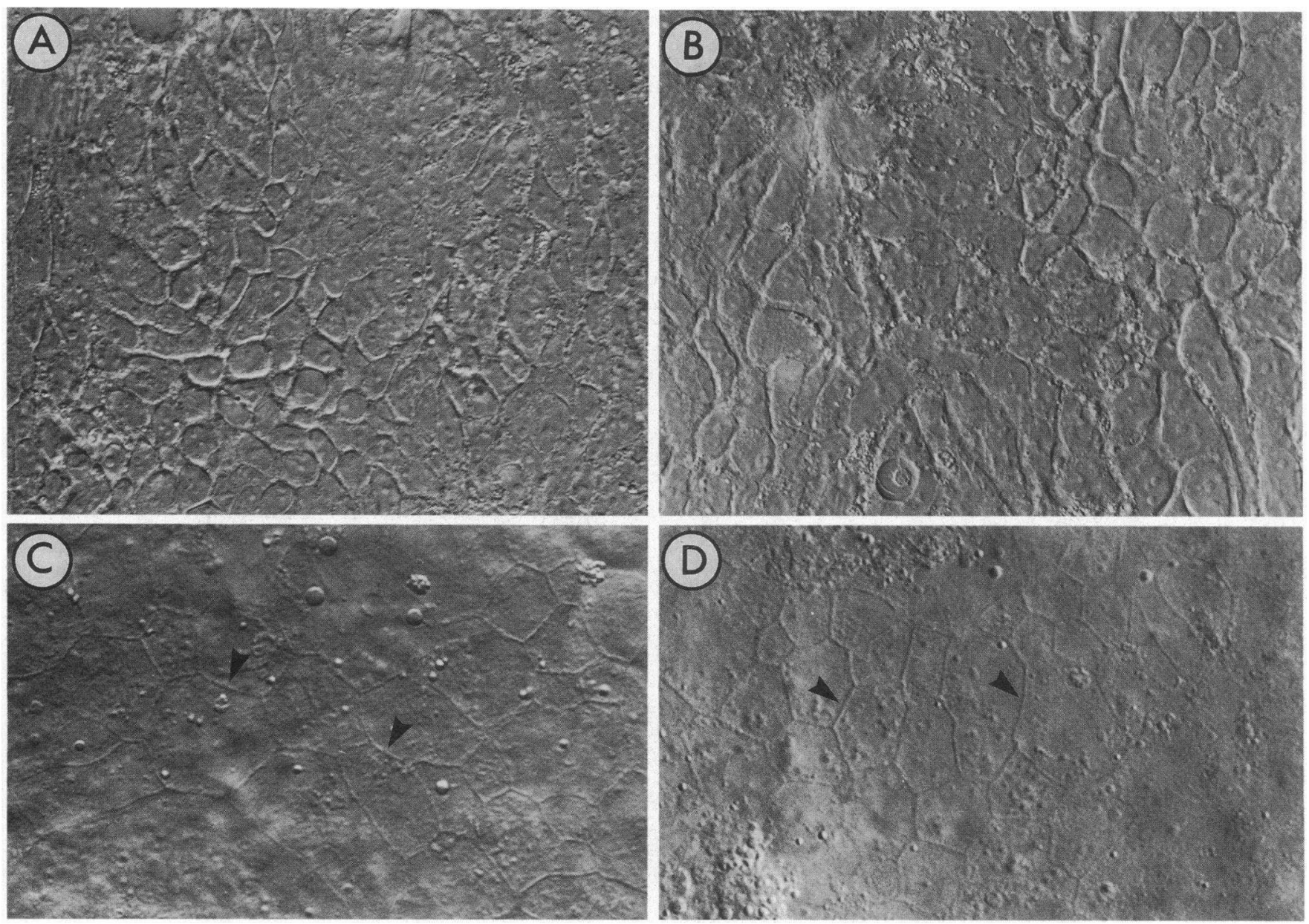

Figure 7. Phase micrographs using Nomarski optics of control and toxin-treated $\left(7 \times 10^{-1} \mu \mathrm{g} / \mathrm{ml}\right.$ for $\left.8 \mathrm{~h}\right) \mathrm{T}_{84}$ cells. $\mathrm{T}_{84}$ monolayers were grown to confluency on glass coverslips, then examined and photographed in the living, unfixed state. $A$ (control) and $B$ (toxin-treated) are photomicrographs taken at the level of the nuclei. The diameters

meation pathways across the tight junction). In the absence of gross disruption of the monolayer (cell death), the magnitude of the toxin-induced alterations in resistance and mannitol flux suggest that the observed changes in tight junction permeability are widespread. That molecules the size of raffinose and inulin remain relatively restricted by these altered junctional pathways indicates that the changes in dimension of this pathway are relatively subtle (i.e., cells are not pulled apart).

Fig. 6 plots resistance against mannitol flux for each discrete flux period after exposure of monolayers to $7 \times 10^{-1}$ $\mu \mathrm{g} / \mathrm{ml}$ toxin A. Nearly maximal resistance alterations occurred before large increments in mannitol flux were seen. Resistance thus is a very sensitive indicator of altered barrier function because major changes in this parameter are induced by relatively minor alterations in mannitol flux.

Morphology. After a 24-h exposure to $7 \times 10^{-1} \mu \mathrm{g} / \mathrm{ml}$ of toxin A, monolayers became disrupted because of scattered areas in which cells were lost. Such cell loss, at these later time points, appeared to be due to cell detachment from the monolayer rather than from cell death as LDH was not spontaneously released into the supernatant, but was dramatically increased after Triton X-100 exposure (data not shown). We did

of toxin-exposed cells are slightly greater than those of controls. $C$ (control) and $D$ (toxin-exposed) are photographed at the level of the apical membrane, allowing visualization of the intercellular junctions (arrowheads). Toxin-treated cells show no evidence of separation from their neighbors.

not further pursue these findings but rather concentrated on monolayers exposed to the above dose of toxin for $8 \mathrm{~h}$, a time at which huge permeability abnormalities occurred in the absence of biochemically detected cell death (Fig. 3 and Table I). $1-\mu \mathrm{m}$ sections of control monolayers showed tall cuboidal to columnar cells with polarized features including basal lipid droplets and nuclei and supranuclear lysosomal vacuoles, as previously described in detail (9). Monolayers exposed to 7 $\times 10^{-1} \mu \mathrm{g} / \mathrm{ml}$ of toxin $\mathrm{A}$ for $8 \mathrm{~h}$ were also confluent and indistinguishable from controls as assessed in $1-\mu \mathrm{m}$ sections (data not shown).

Cell shape and confluency of control and toxin-exposed monolayers plated on glass coverslips were further characterized using Nomarski optics, an optical technique that allows one to view the monolayer en face and to focus in discrete planes within it (see below). In these studies, living unfixed cells were examined and photographed. As shown in Fig. 7, this technique confirmed our impressions, derived from sec-. tioned monolayers, that cells were confluent. The images shown in Fig. 7, $A$ and $B$ are obtained at the level of the nuclei. In general, it appeared, as is suggested in these figures, that the diameters of toxin A-exposed cells were slightly greater than 

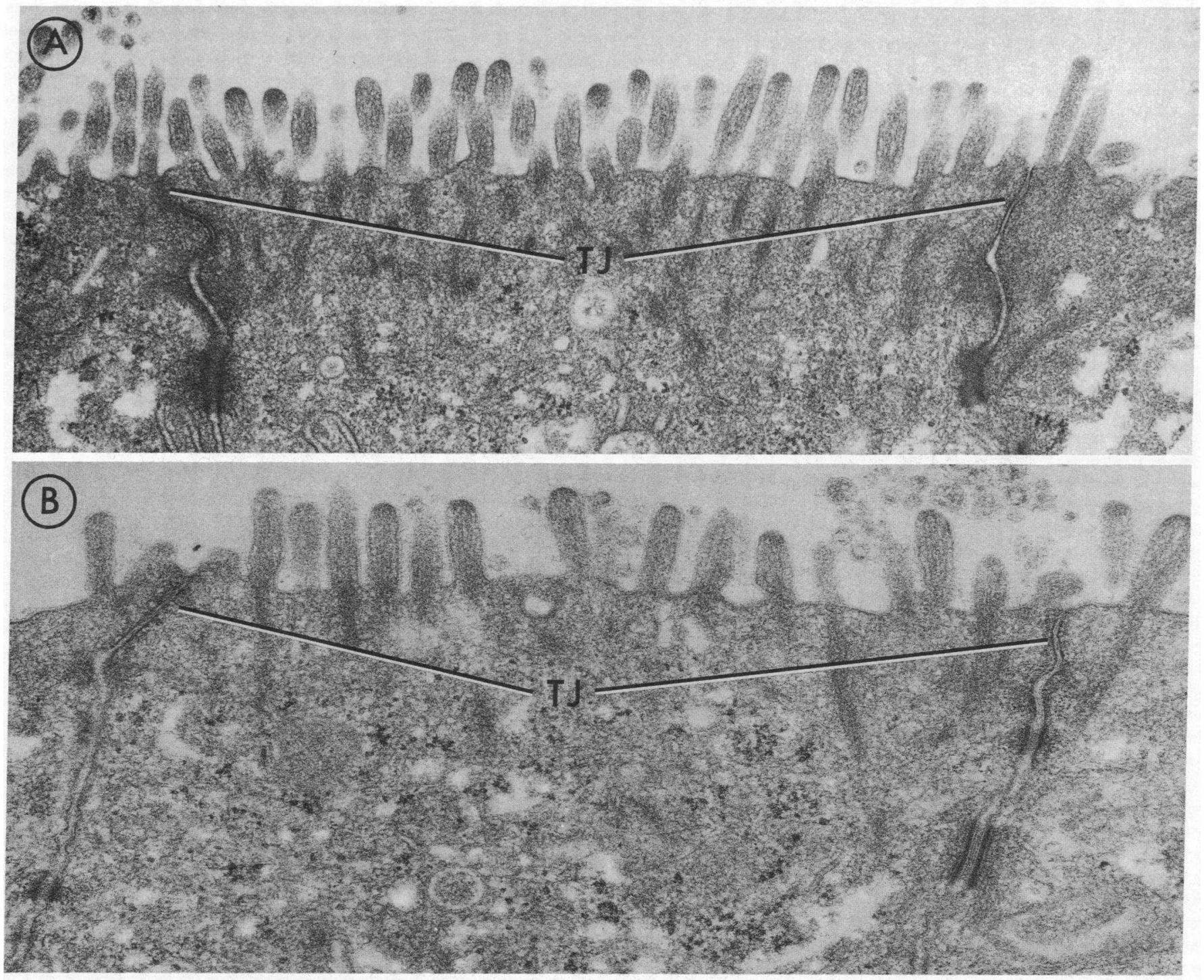

Figure 8. Electron micrographs of $(A)$ control and $(B)$ toxin A-exposed $\left(7 \times 10^{-1} \mu \mathrm{g} / \mathrm{ml}\right.$ for $\left.8 \mathrm{~h}\right) \mathrm{T}_{84}$ monolayers. Although no substantial ultrastructural differences are noted between control and toxin-treated cells, it is of specific interest that the intercellular tight junctions (TJ) of toxin-exposed cells are not grossly separated.

those of controls. The images shown in Fig. 7, $C$ and $D$ are taken from a plane at the level of the apical membrane and thus the area of the intercellular junction can be visualized in the living state. As can be seen, even when large junctional areas (relative to the samples obtained by standard sectioning techniques) are examined, toxin A-treated cells show no evidence of separation from their neighbors.

Electron micrographs of routine thin sections also failed to show substantial ultrastructural differences between control (Fig. $8 \mathrm{~A}$ ) and toxin-treated $(8 \mathrm{~h}$, above dose, Fig. $8 \mathrm{~B}$ ) monolayers. Of specific interest was the finding that tight junctions of toxin-treated monolayers were not grossly disrupted (Fig. 8 $B)$. The functional findings presented previously, which show substantial toxin A-induced junctional permeability abnormalities in the absence of cell death, thus cannot be explained by gross separation of cells from their neighbors. Rather, these data indicate that subtle abnormalities in tight junctions, which are beyond the resolution of these standard electron micrograph (EM) techniques, are present at this stage. Such standard EM techniques are not ideal for studies of the state of cytoskeletal elements, such as microfilaments. This is because microfilaments may pass through the plane of section and also because the background electron density due to the presence of soluble cytoplasmic proteins obscures the image of fine cytoskeletal structures. Either detergent extraction (to clear cytoplasmic soluble proteins) or fluorescent localization techniques (used here and described below) thus are more sensitive for this purpose.

To determine whether an alteration in filamentous actin $(F$ actin) distribution or density might parallel the observed functional changes, fluorescent staining of $F$ actin with rhodamine-labeled phalloidin was performed. Photomicrographs obtained with 1-s film exposure times showed that, in control monolayers, $F$ actin was localized as a prominent perijunctional peripheral ring with a fine, flocculant pattern of staining occurring within the cell body (Fig. $9 \mathrm{~A}$ ). In striking contrast, 

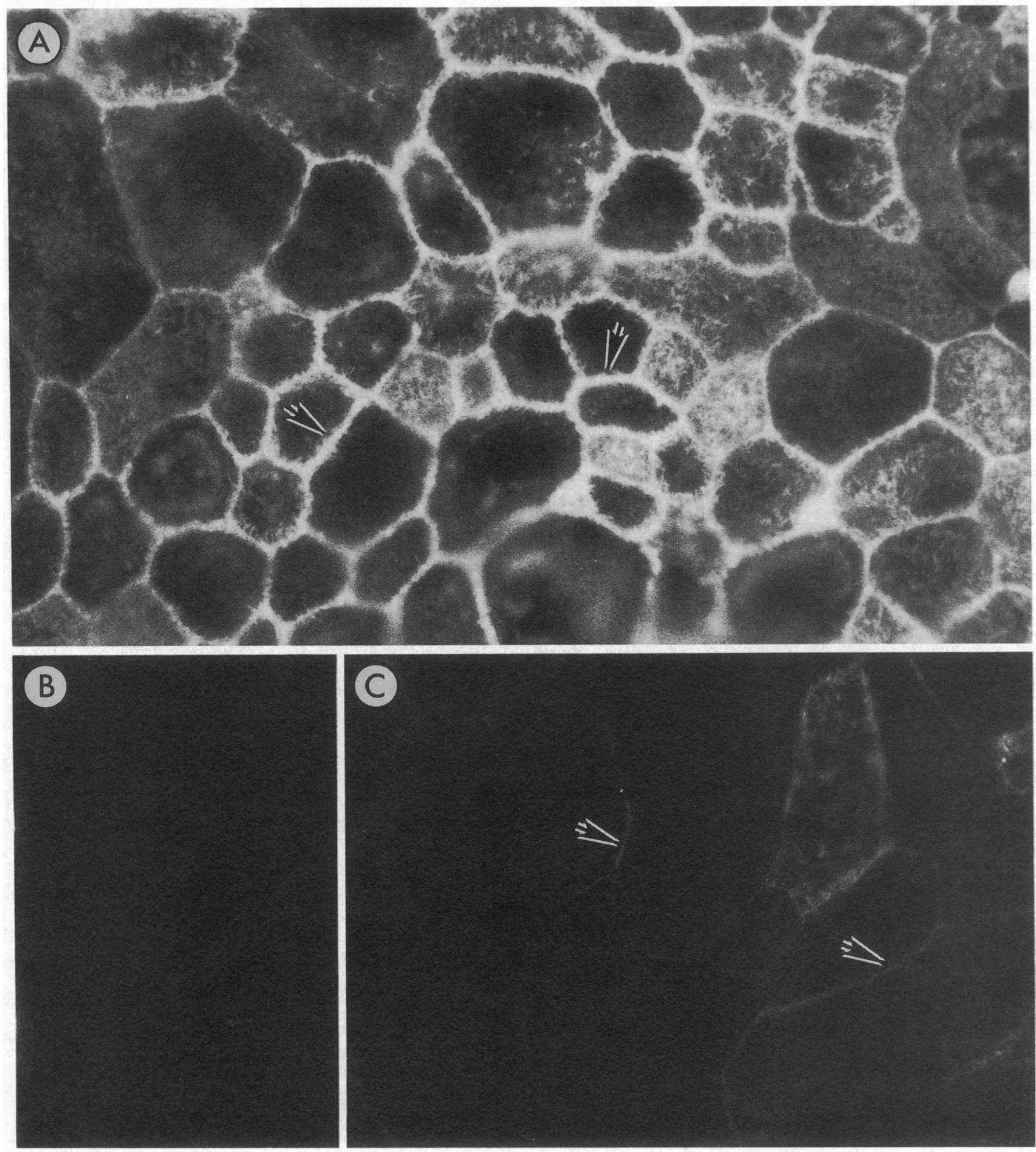

Figure 9. Photomicrographs of $(A)$ control and $(B$ and $C$ ) toxin-exposed $\left(7 \times 10^{-1} \mu \mathrm{g} / \mathrm{ml}\right.$ for $\left.8 \mathrm{~h}\right) \mathrm{T}_{84}$ monolayers fluorescently stained for $\mathrm{F}$ actin with rhodamine-labeled phalloidin. $A$ and $B$ were both taken with a 1-s film exposure time. $(A)$ Control monolayers show that $\mathrm{F}$ actin is localized as a prominent perijunctional ring (arrows) with a fine, flocculant staining within the cell body. In striking contrast, photomicrographs of monolayers treated with toxin A show es- sentially no fluorescent staining when photographed with the same 1-s exposure $(B)$. (C) An 8-s exposure of the same field, however, demonstrates the presence of cells but the perijunctional ring (arrows) is barely visible and staining within the cell borders is nearly absent. Again, the diameters of toxin-treated cells $(C)$ appear greater than those of control cells $(A)$. 
photomicrographs (1-s film exposure time) of monolayers treated with $7 \times 10^{-1} \mu \mathrm{g} / \mathrm{ml}$ of toxin A for $8 \mathrm{~h}$ showed much less staining (Fig. $9 \mathrm{~B}$ ). An 8-s exposure of the same field revealed that cells and rings were indeed present but that staining was markedly diminished (Fig. $9 C$ ).

\section{Discussion}

The first major finding of this study is that $C$. difficile toxin A can exert a direct effect on a model intestinal epithelium in the absence of mesenchymal, endothelial, or inflammatory cells. This finding is of potential importance, as it has variously been suggested that the intestinal lesions associated with pseudomembranous colitis in humans may be due to a primary endothelial effect (5) and that the toxin A effects on rabbit ileum are secondary to toxin-stimulated release of mediators from inflammatory cells (6). The most striking effect of toxin $A$ on $T_{84}$ monolayers described here is markedly diminished barrier function, as indicated by a huge decrease in transepithelial resistance. This effect is not due to cell death or nonspecific leakage of cell membranes to small molecules (mannitol efflux and $\mathrm{LDH}$ and ${ }^{51} \mathrm{Cr}$ data), to gross separation of cells, or to detachment of epithelial cells (morphology data) with disruption of the monolayer. Rather, the barrier defect elicited by toxin A appears to be primarily attributable to an alteration in tight junction permeability. Moreover, the size of the tight junction defect elicited by toxin $A$ is such that while junctional permeability to ions and hydrophilic hexose-sized molecules is markedly increased, permeability to hydrophilic molecules with hydrodynamic radii of $5.7 \AA$ or greater is relatively unaffected. Our morphological studies indicate that substantially longer exposures to toxin A result in disruption of monolayer confluency, a condition that would obviously be associated with loss of the epithelial barrier to macromolecules. However, even the limited permeability abnormalities seen with toxin $\mathrm{A}$ exposures of 4-8 h might have profound influences on epithelial function. The primary function of intestinal epithelium is the vectorial transport of ions and small nutrients. The success of this process depends, in part, on the ability of the tight junction to separate the fluid compartments that interface with the apical and basolateral membranes. Additionally, such barrier defects, even if limited in size, could potentially enhance the transepithelial movement of small chemotactic peptides that are produced by bacterial species inhabiting the intestinal lumen (17). Even in the presence of a confluent epithelium, toxin A-induced barrier defects thus might stimulate migration of PMN out of vessels and into the intestinal epithelium, and activation of these inflammatory cells would ensue. Whereas these latter possibilities are entirely speculative, they highlight the physiological significance that even limited defects in junctional barrier function might have.

The second major observation reported here is that exposure of this model intestinal epithelium to toxin A can result in dramatic alteration in cytoskeletal structure. Specifically, F actin becomes redistributed but even more impressive, $F$ actin staining is markedly diminished after exposure to toxin A, which may be responsible for the functional impairment described above. Previously, only toxin B had been shown to have effects on cytoskeletal structure and these effects were described in cell types not of intestinal epithelial origin (18, 19). It is interesting to us that these studies showed that toxin B causes major alterations of $F$ actin distribution $(18,19)$ and decreases in the cellular content of F actin (20). Our results suggest that similar effects occur in intestinal epithelial cells exposed to toxin A. These findings cannot be explained by contamination of our toxin A preparations with toxin B, as the toxin A preparation we used was highly purified and pilot observations indicate that, dose for dose, toxin B is less effective in producing permeability abnormalities in $\mathrm{T}_{84}$ cell monolayers than toxin A (Hecht, G., unpublished observation).

Our studies show that toxin A exposure has striking effects on at least one component of the cytoskeleton, filamentous actin. This effect cannot be attributable to toxin $A$ interfering with the binding of phalloidin to $\mathrm{F}$ actin, as the toxin concentration used for these studies when mixed directly with rhodamine phalloidin did not alter the staining of control $\mathrm{T}_{84}$ monolayers (data not shown). In other data not shown, we were unable to mimic the cytoskeletal effects of toxin A by using 8-bromo-cAMP or $\mathrm{Ca}^{2+}$ ionophore A23187 in concentrations known to elicit secretion in $\mathrm{T}_{\mathbf{8 4}}$ cells. These findings suggest that the toxin A effects are unlikely to be due to release of either of these mediators. The parallel occurrence of alterations in cytoskeletal structure and tight junction permeability after toxin A exposure is of interest. We $(16,21)$ and others $(22,23)$ have shown that pharmacological manipulation of the cytoskeleton of epithelial cells often results in altered tight junction permeability and/or structure. Agents that are most potent in this response are those that, while having multiple effects, generally are thought to directly affect actin or interfere with actin-dependent interactions with other cytoskeletal components. These observations apply to both native intestinal epithelia $(16,21)$ and $T_{84}$ monolayers (10). Indeed, we have recently shown that in native intestinal epithelia, cytoskeletal components associate with the lateral membrane directly at the site of the tight junction (24). Moreover, we have shown that physiological (25) manipulation of elements of the perijunctional cytoskeleton also result in alterations in tight junction structure and function. In light of these facts, these data entice us to speculate that the effects of toxin $A$ on $T_{84}$ cell junctional permeability may be secondary to the cytoskeletal effects produced by this toxin.

\section{Acknowledgments}

The authors thank Ms. Virginia Hamel for her efforts in the preparation of this manuscript and Ms. Joan Stafford and Susan Carlson for expert technical support.

This work was supported by National Institutes of Health grants DK-35932 and DK-34854. Dr. Hecht is supported by National Research Service Institutional Training Program DK-07533 and National Research Service Award DK-08020. Dr. Pothoulakis is an American Gastroenterological Association/Glaxo Research Scholar.

\section{References}

1. Taylor, N. S., G. M. Thorne, and J. G. Bartlett. 1981. Comparison of two toxins produced by Clostridium difficile. Infect. Immun. 34:1036-1043.

2. Lyerly, D. M., D. E. Lockwood, S. H. Richardson, and T. D. Wilkins. 1982. Biological activities of toxins A and B of Clostridium difficile. Infect. Immun. 35:1147-1150.

3. Donta, S. T., N. Sullivan, and T. D. Wilkins. 1982. Differential effects of Clostridium difficile toxins on tissue-cultured cells. J. Clin. Microbiol. 15:1157-1158. 
4. Chang, T. W., M. Lauermann, and J. G. Bartlett. 1979. Cytotoxicity assay in antibiotic-associated colitis. J. Infect. Dis. 140:765-770.

5. Price, A. B., and D. R. Davies. 1977. Pseudomembranous colitis. J. Clin. Pathol. 30:1-12.

6. Triadafilopoulos, G., C. Pothoulakis, M. J. O'Brien, and J. T. LaMont. 1987. Differential effects of Clostridium difficile toxins $A$ and B on rabbit ileum. Gastroenterology. 93:273-279.

7. Rothman, S. W., J. E. Brown, A. Diecidue, and D. A. Foret. 1984. Differential cytotoxic effects of toxins A and B isolated from Clostridium difficile. Infect. Immun. 46:324-331.

8. Madara, J. L., and K. Dharmsathaphorn. 1985. Occluding junction structure-function relationships in a cultured epithelial monolayer. J. Cell Biol. 101:2124-2133.

9. Madara, J. L., J. Stafford, K. Dharmsathaphorn, and S. Carlson. 1987. Structural analysis of a human intestinal epithelial cell line. Gastroenterology. 92:1133-1145.

10. Madara, J. L., J. Stafford, D. Barenberg, and S. Carlson. 1988. Functional coupling of tight junctions and microfilaments in $\mathrm{T}_{\mathbf{8 4}}$ monolayers. Am. J. Physiol. 254:G416-G423.

11. Sullivan, N. M., S. Pellett, and T. D. Wilkins. 1982. Purification and characterization of toxins $\mathrm{A}$ and $\mathrm{B}$ of Clostridium difficile. Infect. Immun. 35:1032-1040.

12. De, S. N., and D. N. Chatterje. 1953. An experimental study on the mechanism of action of Vibrio cholerae on the intestinal mucus membrane. J. Pathol. Bacteriol. 66:559-562.

13. Dharmsathaphorn, K., J. A. McRoberts, K. G. Mandel, L. D. Tisale, and H. Mesui. 1984. A human colonic tumor cell line that maintains vectorial electrolyte transport. Am. J. Physiol. 246 (Gastrointest. Liver Physiol. 9):G204-G208.

14. Jarett, L. 1980. Gradwohl's Clinical Laboratory Methods and Diagnosis. Vol. 1. C. V. Mosby Co., St. Louis, MO. 319.

15. Dawson, D. C. 1977. $\mathrm{Na}$ and $\mathrm{Cl}$ transport across the isolated turtle colon: parallel pathways for transmural ion movement. $J$. Membr. Biol. 37:213-233.

16. Madara, J. L., D. Barenberg, and S. Carlson. 1986. Effects of cytochalasin D on occluding junctions of intestinal absorptive cells: further evidence that the cytoskeleton may influence paracellular permeability and junctional charge selectivity. J. Cell Biol. 102:21252136.

17. Marasco, W. A., S. H. Phan, H. Krutzsch, H. J. Showell, D. E. Feltner, R. Nairn, E. L. Becker, and P. A. Ward. 1984. Purification and identification of formyl-methionyl-leucyl-phenylalanine as the major peptide neutrophil chemotactic factor produced by $E$. Coli. J. Biol. Chem. 259:5430-5439.

18. Thelestam, M., and M. Bronnegard. 1980. Interaction of cytopathogenic toxin from Clostridium difficile with cells in tissue culture. Scand. J. Infect. Dis. (Suppl.) 22:16-29.

19. Wedel, N., P. Toselli, C. Pothoulakis, B. Faris, P. Oliver, C Franzblau, and T. LaMont. 1983. Ultrastructure effects of Clostridium difficile toxin B on smooth muscle cells and fibroblasts. Exp. Cell Res. 148:413-422.

20. Pothoulakis, C., L. M. Barone, R. Ely, B. Faris, M. E. Clark, C. Franzblau, and J. T. LaMont. 1986. Purification and properties of Clostridium difficile cytotoxin B. J. Biol. Chem. 261:1316-1321.

21. Madara, J. L., R. Moore, and S. Carlson. 1987. Alteration of intestinal tight junction structure and permeability by cytoskeletal contraction. Am. J. Physiol. 253:C854-C861.

22. Bentzel, C. H., B. Hainan, S. Ho, S. W. Hui, A. Edelman, T. Anagnostopoulos, and E. L. Beneditt. 1980. Cytoplasmic regulation of tight-junction permeability: effect of plant cytokinins. Am. J. Physiol. 239 (Cell Physiol. 8):C75-C89.

23. Cereijido, M., I. Meza, and A. Martinez-Palomo. 1981. Occluding junctions in cultured epithelial monolayers. Am. J. Physiol. 240 (Cell Physiol. 9):C96-C102.

24. Madara, J. L. 1987. Intestinal absorptive cell tight junctions are linked to cytoskeleton. Am. J. Physiol. 253 (Cell Physiol. 22):C171C175.

25. Madara, J. L., and J. R. Pappenheimer. 1987. The structural basis for physiological regulation of paracellular pathways in intestinal epithelia. J. Membr. Biol. 100:149-167. 